\title{
Multiscale Diffusion Method for Simulations of Long-Time Defect Evolution with Application to Dislocation Climb
}

\author{
K. L. Baker ${ }^{\mathrm{a}, *}$ W. A. Curtin ${ }^{\mathrm{a}}$ \\ ${ }^{a}$ Institute of Mechanical Engineering, EPFL, CH-1015 Lausanne, Switzerland
}

\begin{abstract}
In many problems of interest to materials scientists and engineers, the evolution of crystalline extended defects (dislocations, cracks, grain boundaries, interfaces, voids, precipitates) is controlled by the flow of point defects (interstitial/substitutional atoms and/or vacancies) through the crystal into the defect. Precise modeling of this behavior requires fully atomistic methods in and around the extended defect, but the flow of point defects entering the extended defect region can be treated by coarse-grained methods. Here, a multiscale algorithm is presented to provide this coupling. Specifically, direct accelerated molecular dynamics (AMD) of extended defect evolution is coupled to a diffusing point defect concentration field that captures the long spatial and temporal scales of point defect motion in the presence of the internal stress fields generated by the evolving defect. The algorithm is applied to study vacancy absorption into an edge dislocation in aluminum where, under a sufficient driving stress and vacancy supersaturation, vacancy accumulation in the core leads to nucleation of a double-jog that then operates as a sink for additional vacancies; this corresponds to the initial stages of dislocation climb modeled with explicit atomistic resolution. The method is general and so can be applied to many other problems associated with nucleation, growth, and reaction due to accumulation of point defects in crystalline materials.
\end{abstract}

Keywords: diffusion, accelerated molecular dynamics, vacancy, dislocation

\section{Introduction}

In many engineering materials, the interaction of mobile point defects (interstitial or substitutional atoms, or vacancies) and extended crystalline defects (dislocations, grain boundaries, voids, interfaces, precipitates) controls the microstructural evolution and mechanical properties of the material. Specific examples across the range of possibilities include precipitate growth, dynamic strain aging, and dislocation climb. These interactions are difficult to model due to the wide range of time and spatial scales involved. With regard to timescales, diffusion of point defects in the bulk usually takes nanoseconds to microseconds for each individual hop among atomic sites while diffusion among atomic sites within an extended defect can be orders of magnitude faster due to the non-crystalline environment in the defect. With regard to spatial scales, point defect concentrations are usually low, so that long-range diffusion is required to accumulate point defects in a local region.

\footnotetext{
${ }^{*}$ Corresponding author

Email address: kristopher.baker@epfl.ch (K. L. Baker )
} 
Furthermore, the diffusion into the defect is affected by the stress fields generated by the extended defect itself, which can be long-ranged in the cases of dislocations and cracks, and these stress fields raise or lower the point defect formation energy at each atomic site. The long-range diffusion of point defects is thus affected by the precise stress fields generated by the extended defect as it evolves.

Typical modeling strategies cannot handle the above diversity of time and length scales. Accelerated molecular dynamics (AMD) can model atomic diffusion $[1,2,3,4,5,6]$ but is limited, for computational reasons, to relatively small volumes and is not amenable to long-range diffusion. There are also no associated methods for injecting or extracting point defects into such an atomistic model. Large scale diffusion in the presence of weak stress gradients can be modeled using the diffusion equation, and discrete diffusion (DD) methods can be used when field gradients are stronger [7] and resolution of the concentration on a site-by-site basis is desired [8]. But these methods are inapplicable in and around extended defects since the underlying migration barriers in the defects are unknown and because the defect structure can be changing as point defects are absorbed. Here, we propose a multiscale method to couple AMD and Discrete Diffusion directly and accurately. The method captures the long-range, long-time diffusion around an evolving defect using DD in the presence of the stress field generated by the defect, the injection of point defects into the extended defect region via algorithms to be described below, and the atomistic evolution of the extended defect as the point defects are absorbed via AMD. The method, which we label DD-MD, is quite general and uses rigorous methods and controllable approximations that enable extended defect evolution in an atomistic simulation.

The rest of this paper is organized as follows. Section 2 describes the coupled DD-MD diffusion algorithm and the numerical methods used within it. Section 3 applies the DD-MD method to study vacancy absorption into an edge dislocation core in aluminum. Concluding remarks are presented in section 4 .

\section{Methods and Algorithms}

This work focuses on the interaction of two classes of defects: atomic-sized point defects and multi-atom extended defects. When far from an extended defect, point defects (e.g. interstitial atoms, substitutional atoms, or vacancies) diffuse through the distorted crystalline lattice. The transition rate of a point defect from one site to another depends on the formation energies at the initial and final site and the migration barrier between them, all of which vary with the local distortion caused by applied loads or eigenstrains of remote defects. Extended defects contain many atoms and include dislocations, cracks, grain boundaries, bi-material interfaces, voids, precipitates, and stacking-fault tetrahedra. Ideal structures of these extended defects can be represented at the atomic scale, but we are interested in the evolving structures due to absorption/interaction with point defects. For specificity, we present the DD-MD algorithm using the specific case of vacancies interacting with an edge dislocation, with the evolution of the ideal edge dislocation ultimately corresponding to jog formation and jog motion, i.e. the dislocation climb process.

The general problem of interest is a large domain subject to both mechanical and chemical boundary conditions, i.e. applied displacements/tractions and imposed concentrations/fluxes, as shown schematically in Figure 1. An extended defect resides inside the domain; in some region around the defect, the atomic distortions are too large for the application of standard point defect diffusion and linear elasticity, and so this region must be treated by explicit AMD. Outside this 
This is a pre-print of the following article: Baker, K. L.; Curtin, W. A. J. Mech. Phys. Solids 2016, 92, 297-312.. The formal publication is available at http://dx.doi.org/10.1016/j.jmps.2016.04.006

region, standard diffusion (see below) and/or coarse-grained mechanics (linear elasticity, finite elements, etc.) can be applied. There is a boundary between the extended defect region and the surrounding domain where mechanical and chemical coupling must be achieved. The mechanical coupling of atoms to continuum is well-established and will not be addressed here [9, 10, 11]; here we use fully-resolved molecular statics in the outer domain and avoid introduction of any multiscale mechanical coupling. Our focus is thus on the chemical coupling, i.e. the flow of point defects between extended defect region and the surrounding domain.

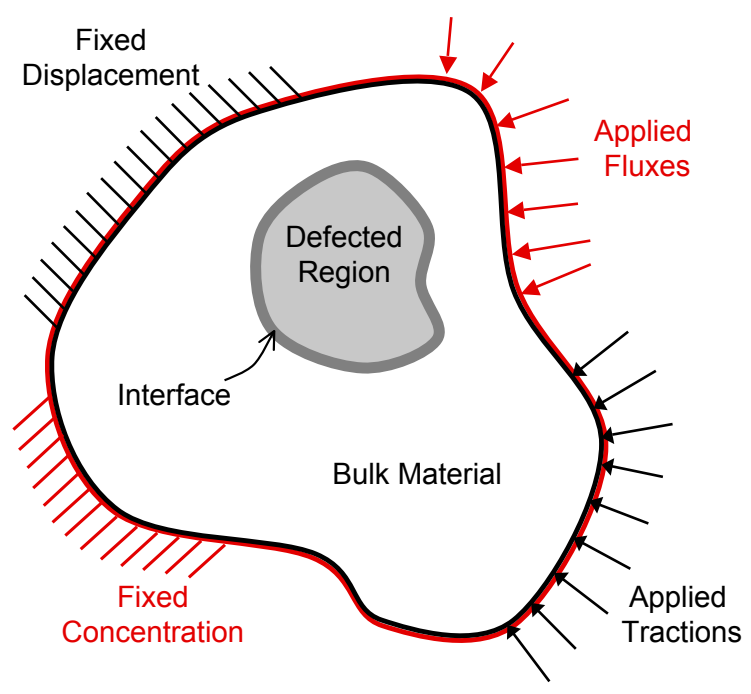

Figure 1: A general schematic of the chemo-mechanical system of interest. Mechanical boundary conditions consisting of prescribed displacements and/or tractions are applied the the outer boundary of the system, along with fixed concentration and/or flux boundary conditions. The model is divided into bulk (white area) and defected (grey area) regions that define which numerical technique is used to model the point defect diffusion. The volume of material that forms the interface between the two regions (dark grey surface) uses both AMD and the diffusion equation so that information can be passed between the two regions.

Within the overall domain, we define different zones as indicated in Figure 2 for the case of an edge dislocation embedded in a large cylindrical geometry. Zone 0 contains the outer boundary of the overall problem where the external boundary conditions are applied. Zone 1 corresponds to the domain where the material is crystalline with small distortions, where the standard diffusion (and continuum mechanics) is applied. Zones 2 and 3 denote two adjacent atomic surfaces where a welldefined (but distorted) crystal structure still exists adjacent to the extended defect, and where the coupling of the DD and AMD methods is achieved. Zone 4 is the domain containing the evolving extended defect and surrounding highly-distorted atomistic regions where AMD is required. As the simulation proceeds, point defects flow into Zone 3 from the surrounding crystal (Zone 2) as dictated by Discrete Diffusion and then those point defects evolve and react with other defects in Zones 3 and 4 as dictated by the AMD simulations. The definitions of Zones 1,2,3, and 4 can evolve adaptively during the evolution; for description of the method we consider them as fixed over some window of time. Diffusion of point defects is executed in Zones 0-3 using Discrete Diffusion since the crystalline sites (substitutional or interstitial) are well defined (Figure 2c). Boundary conditions for 
This is a pre-print of the following article: Baker, K. L.; Curtin, W. A. J. Mech. Phys. Solids 2016, 92, 297-312.. The formal publication is available at http://dx.doi.org/10.1016/j.jmps.2016.04.006

sites in Zone 0 are imposed. Boundary conditions for sites $i$ in Zone 3 are determined in part by the concentration in Zone 3 that comes from the AMD simulation containing $n$ point defects, $c_{i}^{(3, M D, n)}$. Accelerated Molecular Dynamics is executed in Zones 2-4 with atoms in Zones 0-1 held fixed at their mechanical equilibrium positions and serving as mechanical boundary conditions (Figure 2d).
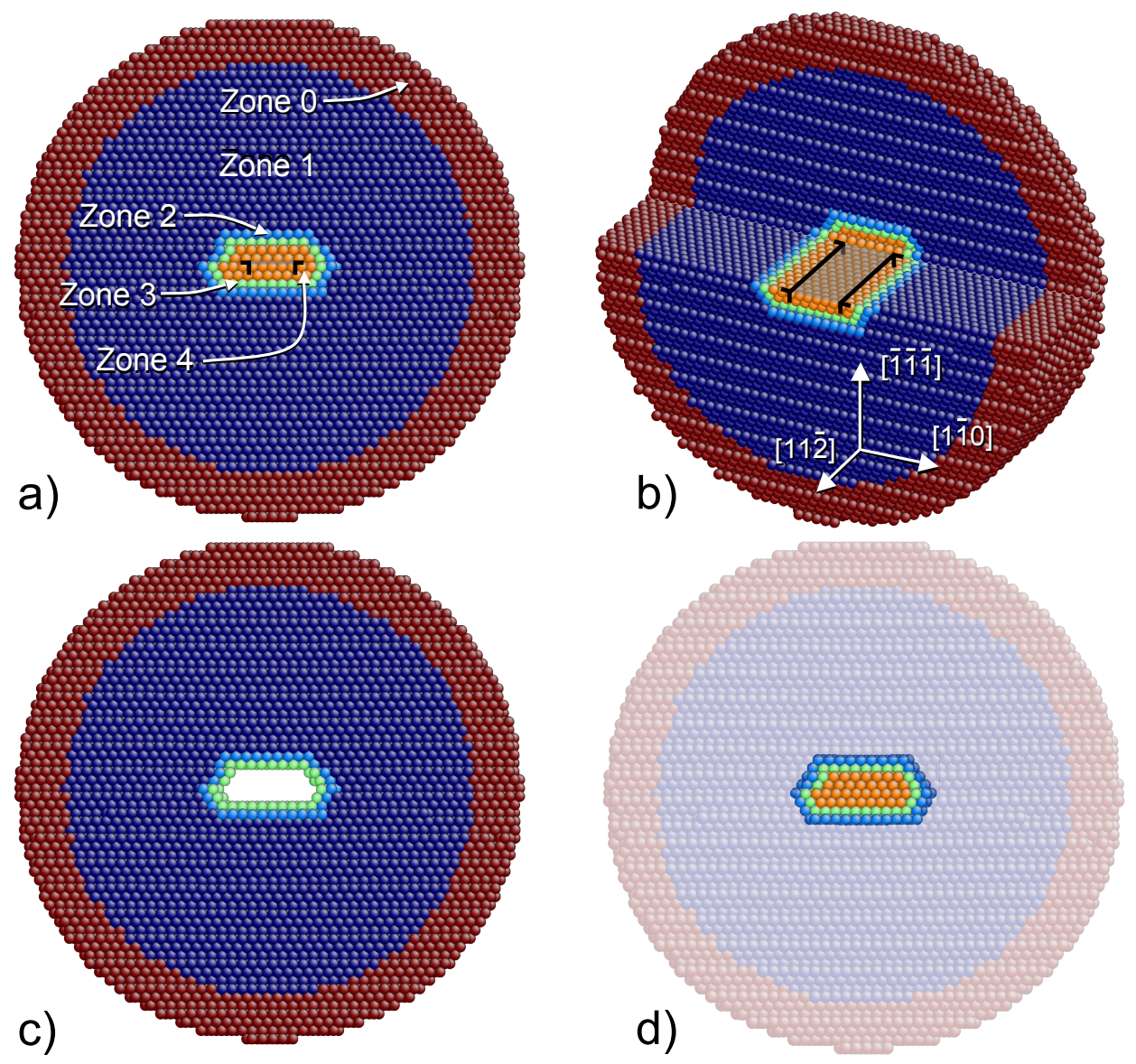

Figure 2: (Color online) Decomposition of entire problem domain into Zones 0-4, for the example of a dissociated edge dislocation in aluminum. The overall domain is a cylindrical volume with the periodic axis along the dislocation line direction. a) Cross section of the simulation volume with the dislocation line perpendicular to the plane of the image, showing Zones 0-4. Zone 4 (orange) contains the two partial dislocation cores and stacking fault (black symbols plus gray line) and surrounding atoms. Zone 3 (green) consists of the shell of atoms surrounding Zone 4 . Zone 2 (light blue) consists of the next shell of atoms surrounding Zone 3. Zone 1 (dark blue) consists of all other outer atoms aside from the boundary atoms in Zone 0 (red). b) Cut-away cross-section of the same system showing the extent along the dislocation line. c) Domain of Zones 0-3 within which the discrete diffusion problem is solved. d) Domain of Zones 2-4 in which the AMD simulation is run, with Zones 0-1 held fixed as mechanical boundary conditions.

The overall DD-MD algorithm operates in a staggered fashion, described succinctly as follows, with all details given subsequently below. We start with $n=0$ defects in Zones 3 and 4 and 
thus $c_{i}^{(3, M D, n=0)}=0$. One point defect is then inserted randomly into the AMD domain (Figure $2 \mathrm{~d}$ ), and an AMD simulation is run until the system achieves an estimated equilibrium in Zones 2-4 with Zones 0-1 held fixed as boundary conditions. From the distribution of point defects in Zone 3 found in the AMD simulation, the concentration of point defects in Zone 3 with $n=1$ is computed as $c_{i}^{(3, M D, n=1)}$. Diffusion in Zones 0-3 is then executed for some time increment with an initial concentration in Zone $3 c_{i}^{(3)}(t)$ that is determined by the AMD concentrations with $n=0,1$ defects, $c_{i}^{(3, M D, n=0)}$ and $c_{i}^{(3, M D, n=1)}$. When the integrated flux of point defects flowing into Zone 3 reaches unity, the actual time $t_{n=1}$ at which there is exactly $n=1$ point defect contained in Zones 3 and 4 (corresponding to the AMD simulation with $n=1$ point defects) has been reached. A second point defect $(n=2)$ is then inserted randomly into the AMD domain, and AMD is run until estimated equilibrium is achieved in Zones 2-4. The concentration of point defects in Zone 3 with $n=2$ is then computed as $c_{i}^{(3, M D, n=2)}$ and diffusion in Zones $0-3$ is then run with evolving initial conditions for $c_{i}^{(3)}(t)$ determined by $c_{i}^{(3, M D, n=1)}$ and $c_{i}^{(3, M D, n=2)}$. The time at which the integrated flux of point defects into Zone 3 has again reached unity is the actual time at which there are $n=2$ point defects contained in Zones 3 and $4, t_{n=2}$. The algorithm continues in the loop of inserting point defects into the AMD domain, running AMD in Zones 2-4, and running diffusion in Zones 0-3. As point defects flow into Zone 3 by the diffusion equation and are distributed into Zones 3 and 4 by AMD, the extended defect structure may evolve and the mechanical state of the entire system of Zones 0-4 is automatically updated to reflect this evolution. Under some conditions, the entire system may reach equilibrium, at which point the extended defect has accommodated some number of point defects but is in equilibrium with the surrounding crystal; the algorithm reaches this point automatically as the flux from Zone 3 into Zone 2 is equal to the flux of Zone 2 into Zone 3. Under other conditions, the extended defect may absorb point defects forever, with the extended defect evolving in structure continuously.

The DD-MD algorithm sketched above requires elaboration of the following key parts: (i) initialization of the entire system and problem; (ii) specifying the diffusion equation that is solved in Zones 0-3; (iii) definition of the evolving initial conditions for the concentrations in Zone 3 emerging from the AMD simulation; (iv) specifying the AMD method; (v) the method of inserting point defects into Zone 4; and (vi) definition of equilibrium during an AMD simulation. We now address these parts of the algorithm in detail.

\subsection{Initialization for Each New Defect Structure}

Before the DD-MD algorithm is run, the formation or solution enthalpy $\Delta H^{f}$ and misfit volume tensor $\boldsymbol{\Delta} \mathbf{V}^{\mathbf{f}}$ of the point defect of interest are computed independently in the perfect crystal at zero temperature and zero pressure. The migration enthalpy $\Delta H^{m}$ and misfit volume tensor in the transition state $\boldsymbol{\Delta} \mathbf{V}^{\mathbf{t}}$ for site-to-site transitions of the point defect in the perfect crystal are also computed at zero pressure and zero temperature. The details concerning how these properties can be found are provided by Olmsted et al. 2004 [12].

The desired geometry of the problem of interest is then constructed in an atomistic simulation using standard methods, with no point defects in the system, and relaxed to mechanical equilibrium. Zones $0-4$ are then defined by computing the local atomic stress tensor $(\boldsymbol{\sigma})$ and common neighbor analysis (CNA). Zone 4 is then identified as the set of atoms not in the bulk crystal (e.g. fcc) environment plus extra shells of atoms surrounding these non-crystalline atoms where the stress gradients are too large to be handled by discrete diffusion [12]. Zone 3 consists of the shell of near-crystalline atoms surrounding Zone 4 . Zone 2 is the shell of atoms surrounding Zone 3 . Zone 
0 consists of the outer atoms where boundary conditions (mechanical and chemical) are applied, and Zone 1 atoms are all other atoms in between Zones 0 and 2. Figure 2 shows this decomposition of the example problem studied here.

\subsection{Diffusion in Zones 0-3}

The diffusion of point defects through the strained crystalline lattice of Zones 0-3 can be efficiently modeled by considering discrete (site-by-site) diffusion (DD) using transition state theory along with the formation and migration enthalpies appropriate to each site or transition [12]. In most problems, the size of Zones 0-3 is hugely larger than that of Zone 4 and the point defect transition rates in Zones 0-3 are much slower than those in Zone 4, so that this efficient diffusion model captures the large length and time scales of the problem. The discrete diffusion equation is based on the Master Equation approach and defines the time rate of change of the point defect concentration/probability at a crystalline site $i, c_{i}$ in terms of the flux of point defects into(out of) $i$ from(to) neighboring sites $j$ with transition rates $W_{j i}\left(W_{i j}\right)$. Expressing the transition rates in terms of thermal activated events following an Arrhenius law, the evolution of any site $i$ in Zones 0-3 is, following notation of Zhang and Curtin [8],

$$
\frac{\partial c_{i}}{\partial t}=\sum_{j=1}^{N_{n}}\left[c_{j}\left(1-c_{i}\right) \nu_{0} \exp \left(-\frac{Q_{j \rightarrow i}}{k_{B} T}\right)-c_{i}\left(1-c_{j}\right) \nu_{0} \exp \left(-\frac{Q_{i \rightarrow j}}{k_{B} T}\right)\right] .
$$

In Eq. 1, $\nu_{0}$ is the attempt frequency of the diffusional hop, $Q_{i \rightarrow j}$ is the activation energy for a point defect at site $i$ moving to site $j, k_{B}$ is Boltzmann's constant, $T$ is the temperature of the system, and the sum is over the $N_{n}$ nearest neighbor sites of a site $i$ within Zones 0-3. Boundary conditions imposed on sites in Zone 0 can either be fixed concentrations, where $c_{i}$ is imposed and not solved using Eq. 1 above, or fixed flux, where an additional term $d c_{i}^{(f l u x)}$ is added to Eq. 1 .

The activation energy is a function of the migration and formation enthalpy barriers, $\Delta H_{i j}^{m}$ and $\Delta H_{i}^{f}$ respectively, associated with sites $i$ and $j$ as

$$
Q_{i \rightarrow j}=\Delta H_{i j}^{m}-\Delta H_{i}^{f}, \quad Q_{j \rightarrow i}=\Delta H_{i j}^{m}-\Delta H_{j}^{f} .
$$

The migration enthalpy barrier between sites $i$ and $j$ is approximated as a linear function of the stress tensor at the transition state, $\boldsymbol{\sigma}^{t}$, as

$$
\Delta H_{i j}^{m}\left(\boldsymbol{\sigma}^{t}\right) \approx \Delta H^{m}-\boldsymbol{\sigma}^{t} \boldsymbol{\Delta} \mathbf{V}^{\mathbf{t}}
$$

where the stress at the transition state, $\sigma^{t}$, is approximated by the average of the stresses at sites $i$ and $j$ as

$$
\sigma^{t}=\frac{\sigma_{i}+\sigma_{j}}{2}
$$

Similarly, the formation enthalpy barrier at site $i$ is approximated as a linear function of the stress at site $i$ and externally applied stress, $\sigma_{a p p}$, as

$$
\Delta H_{i}^{f}\left(\boldsymbol{\sigma}_{\boldsymbol{i}}, \boldsymbol{\sigma}_{\text {app }}\right) \approx \Delta H^{f}-\boldsymbol{\sigma}_{\boldsymbol{i}} \boldsymbol{\Delta} \mathbf{V}^{\mathrm{f}}-\frac{\operatorname{Tr}\left(\boldsymbol{\sigma}_{\boldsymbol{a p p}}\right)}{3} v_{a}
$$

where $v_{a}$ is the volume per atom in the bulk at zero pressure and zero temperature. The accuracy of the linear approximations for the formation and migration barriers has been demonstrated previously [12]. 
The Discrete Diffusion equation is solved during the time interval $t_{n}<t<t_{n+1}$, i.e. the time interval between the times at which $n$ and $n+1$ point defects have entered Zone 3 from Zone 2 . Within this interval, the diffusion equation is solved over sub-intervals from $t$ to $t^{\prime}=t+\Delta t$. At the start of the sub-interval, the concentration in Zone $3, c_{i}^{(3)}(t)$ is obtained from the concentrations $c_{i}^{(3, M D, n)}$ and $c_{i}^{(3, M D, n+1)}$ as described in the next section. During the sub-interval, the diffusion equation is solved using an embedded 4th- and 5th-order Runge-Kutta method [13]. Starting with a test increment $\Delta t^{(t e s t)}$, this method computes 4 th- and 5th-order estimates of the concentrations in each site at $t^{\prime}=t+\Delta t^{(t e s t)}$. The error between the two estimates is then computed at each site, and the maximum time step $\Delta t^{(\max )}$ that would ensure a maximum absolute error of $1 \times 10^{-6}$ or less is estimated. If $\Delta t^{(\text {test })}<\Delta t^{(\max )}$ then the 5th-order solution is accepted. If $\Delta t^{(\text {test })}>\Delta t^{(\max )}$ then the time step is reduced to $\Delta t^{(t e s t)}=\Delta t^{(\max )}$ and the concentrations are recomputed. A new $\Delta t^{(\max )}$ is then computed, and if smaller than the previous value, the time step is reduced by a factor of 10 , and the concentrations are again recomputed. The final value of $\Delta t^{(\max )}$ is then used as the initial $\Delta t^{(t e s t)}$ for the next time step.

\subsection{Coupling: Boundary Conditions in Zone 3}

At the start of each sub-interval at time $t$, the site concentrations $c_{i}^{(3)}(t)$ are initialized by using a combination of (i) the net flux into Zone 3 from Zone 2 and (ii) the concentrations in Zone 3 emerging from the AMD computations. After diffusion has been run to time $t^{\prime}$, the site concentrations in Zone 3 computed by Discrete Diffusion are $c_{i}^{(3, D D)}\left(t^{\prime}\right)$ where the superscript $D D$ indicates that these concentrations come from the Discrete Diffusion equation. The total number (fraction) of point defects that have entered Zone 3 from Zone 2 since $t_{n}$, the time at which there were $n$ point defects in Zones 3 and 4 , is

$$
\Delta N_{3}\left(t^{\prime}-t_{n}\right)=\sum_{i}\left(c_{i}^{(3, D D)}\left(t^{\prime}\right)-c_{i}^{(3)}\left(t_{n}\right)\right)
$$

If $\Delta N_{3}\left(t^{\prime}-t_{n}\right)<1.0$ then $t^{\prime}$ is less than the time $t_{n+1}$. The site concentrations in Zone 3 at $t^{\prime}$ are then interpolated from the Zone 3 site concentrations derived from the AMD simulations corresponding to $n$ and $n+1$ point defects at times $t_{n}$ and $t_{n+1}$. We use a linear interpolation

$$
c_{i}^{(3)}\left(t^{\prime}\right)=c_{i}^{(3, M D, n)}\left(1-\Delta N_{3}\left(t^{\prime}-t_{n}\right)\right)+c_{i}^{(3, M D, n+1)} \Delta N_{3}\left(t^{\prime}-t_{n}\right) .
$$

This new value for the concentrations in Zone 3 is then used as the initial condition for the next time increment in the Discrete Diffusion evolution. If $N_{3}\left(t^{\prime}-t_{n}\right) \geq 1.0$ at time $t^{\prime}$, then $t^{\prime}$ has exceeded $t_{n+1}$. A new AMD simulation containing $n+2$ point defects is then executed to obtain $c_{i}^{(3, M D, n+2)}$, and the Discrete Diffusion algorithm is then executed with site concentrations in Zone 3 interpolated between the AMD site concentrations measured at times $t_{n+1}$ and $t_{n+2}$, starting with

$$
c_{i}^{(3)}\left(t^{\prime}\right)=c_{i}^{(3, M D, n+1)}\left(2-\Delta N_{3}\left(t^{\prime}-t_{n}\right)\right)+c_{i}^{(3, M D, n+2)}\left(\Delta N_{3}\left(t^{\prime}-t_{n}\right)-1\right) .
$$

In addition, the atomic positions and stresses in Zones 0-3 are updated to those corresponding to the minimum energy configuration discovered during the AMD simulation with $n+1$ point defects. To do so, we start with the minimum energy configuration when $n+1$ defects are present, as found from the AMD simulation, and find the corresponding zero temperature minimum energy structure 
for the entire system of Zones 1-4, given the mechanical boundary conditions of Zone 0. The atomic stresses in Zones 0-3 are then updated using this new structure, with corresponding changes to the activation and formation enthalpies in Zones 0-3. The zero-temperature atomic structure in Zones $0-3$ is then scaled to the finite temperature lattice constant, and this structure provides the mechanical boundary conditions for the AMD simulation of Zones 2-4 with $n+2$ defects. Thus, the entire problem evolves as the extended defect evolves.

\subsection{Accelerated Molecular Dynamics in Zones 2-4: Method, Insertion, Reaching Equilibrium}

Hyperdynamics is applied to accelerate the diffusive motion of the point defects in Zones 2-4 so that the complex system (extended defect plus absorbed point defects) explores the dominant region of phase space. Hyperdynamics biases the dynamics so that activated states are explored very frequently $[5,14]$, as compared to standard MD. To bias the system dynamics in this work, we have implemented the self-learning simplified bond-boost potential of Perez and Voter [2] (see Appendix A for our specific implementation), based on original work by Miron and Fichthorn [1]. The bond-boost potential is based on the bond breaking picture of deformation and assumes that bonds must stretch or compress beyond a certain threshold in order for a transition to occur. Before using the bond-boost hyperdynamics potential, one must choose which atoms receive the bond-boost potential and measure the average bond lengths between all boosted atoms. Here, bonds are built between all pairs of nearest neighbor atoms in Zones 2-4 that contain at least one atom that is not in FCC stacking as determined by the atom's CNA.

An AMD simulation corresponds to a simulation of Zones 2-4 around the extended defect containing $n$ additional point defects in the AMD domain. At the start of the simulation, all atoms are in their mechanical equilibrium position corresponding to the minimum energy state of the system containing $n-1$ point defects and expanded to the finite- $T$ lattice constant. Zone 0 and 1 atomic sites are held fixed and provide boundary conditions for the dynamic simulation. Zone 2 atoms are included in the dynamic AMD simulation so that sites in Zone 3 see dynamic neighbors in Zone 2; otherwise, the probability of finding the point defects in Zone 3 sites is unduly affected. Before the dynamics is started, the positions of possible point defects sites in Zones 2 and 3 are recorded. The $n^{\text {th }}$ point defect is then inserted into Zone 4 (for a vacancy, by randomly deleting one of the Zone 4 atoms) and the energy is minimized using molecular statics holding Zones 0-1 at the expanded lattice. The bond lengths at the resulting minimum energy configuration are used as the average bond lengths for the bond-boost method until the next minimum energy configuration is found.

During AMD, the system of atoms in Zones 2-4 evolves from one minimum energy basin to another via thermally-activated transitions. Information is collected for each basin visited. The potential energy minimum $E_{j}$ of the $j^{\text {th }}$ basin is computed via molecular statics at the expanded lattice constant. The total number of point defects in Zones 2 and $3, m_{j}^{(2)}$ and $m_{j}^{(3)}$, in the $j^{t h}$ basin is also recorded. Once there is a transition out of the $j^{\text {th }}$ basin into another basin, the real time $t_{j}$ spent in the $j^{t h}$ basin is computed using the standard hyperdynamics procedure.

As the AMD simulation continues, more energy basins are explored. The AMD simulation must be run until enough basins have been sampled to approach the equilibrium distribution of states. To assess the approach to equilibrium, we create two distinct estimates of the underlying probability distribution $p(E)$ of the total potential energy. Both distributions are discretized into bins of width $k_{B} T$ because this is a natural energy scale at the operating temperature and is related to range of accessible energy states of the system. The first estimate of the probability distribution, denoted as $p^{\text {count }}\left(E_{i}\right)$, is simply the probability (fraction) of basins having minimum energy in bin $i$, i.e. $E_{i} \leq E_{j}<E_{i}+k_{B} T$. Over the range of basins starting with $j=j_{0}$ and ending with $j=j_{f}$, 
This is a pre-print of the following article: Baker, K. L.; Curtin, W. A. J. Mech. Phys. Solids 2016, 92, 297-312.. The formal publication is available at http://dx.doi.org/10.1016/j.jmps.2016.04.006

the probability $p\left(E_{i}\right)$ of encountering any basin in the $i^{\text {th }}$ energy bin is the total number of energy basins in the interval $\left[E_{i}, E_{i}+k_{B} T\right)$ divided by the total number of basins explored $j=j_{0}, \ldots j_{f}$,

$$
p^{\text {count }}\left(E_{i}\right)=\frac{1}{\sum_{j=j_{0}}^{j_{f}} 1} \sum_{\substack{j=j_{0} \\ E_{j} \in\left[E_{i}, E_{i}+k_{B} T e V\right)}}^{j_{f}} 1
$$

The second estimate of the probability distribution is based on the average number of basins explored between repeat visits to the same energy bin. Given an infinitely long, positive recurrent Markov chain drawn from the underlying probability distribution $p\left(E_{i}\right)$, the probability of being in an energy bin $i$ is given by the inverse of the average number of basins visited between repeat visits to bin $i$ [15]. This estimate of the probability distribution, denoted as $p^{\text {return }}\left(E_{i}\right)$, is calculated as the simulation proceeds by computing the number of basins explored between every return visit to each bin, then computing the average across the number of return visits.

Near-equilibrium is defined here as the point at which the two probability distribution estimates converge to each other, with some additional constraints to ensure good overall sampling statistics. We thus impose three criteria for convergence: at least 1000 basins are explored, the number of basins explored divided by the number of non-zero energy bins must be greater than 100 , and the distance between the distributions as defined by the Hellinger distance metric [15] must be less than 0.10 for 500 consecutively explored basins. The Hellinger distance between the two distributions is computed as

$$
H\left(p^{\text {count }}, p^{\text {return }}\right)=\sqrt{\sum_{i=1}^{N_{\text {bins }}}\left(\sqrt{p^{\text {count }}\left(E_{i}\right)}-\sqrt{p^{\text {return }}\left(E_{i}\right)}\right)^{2}},
$$

where $N_{\text {bins }}$ is the number of bins that have a non-zero probability in either $p^{\text {count }}$ or $p^{\text {return }}$. When all of the criteria are met, the distribution is deemed to have reached near-equilibrium. True equilibrium is never obtained but the AMD has sampled a large collection of basins sufficient to approximate equilibrium. As an illustration, Figure 3 shows the Hellinger distance and the corresponding final binned "near-equilibrium" distributions $p^{\text {count }}$ and $p^{\text {return }}$ for three AMD simulations of a dislocation core containing $n=1,3$ and 5 vacancies, respectively, which will be described in detail in the Application section. 
This is a pre-print of the following article: Baker, K. L.; Curtin, W. A. J. Mech. Phys. Solids 2016, 92, 297-312.. The formal publication is available at http://dx.doi.org/10.1016/j.jmps.2016.04.006
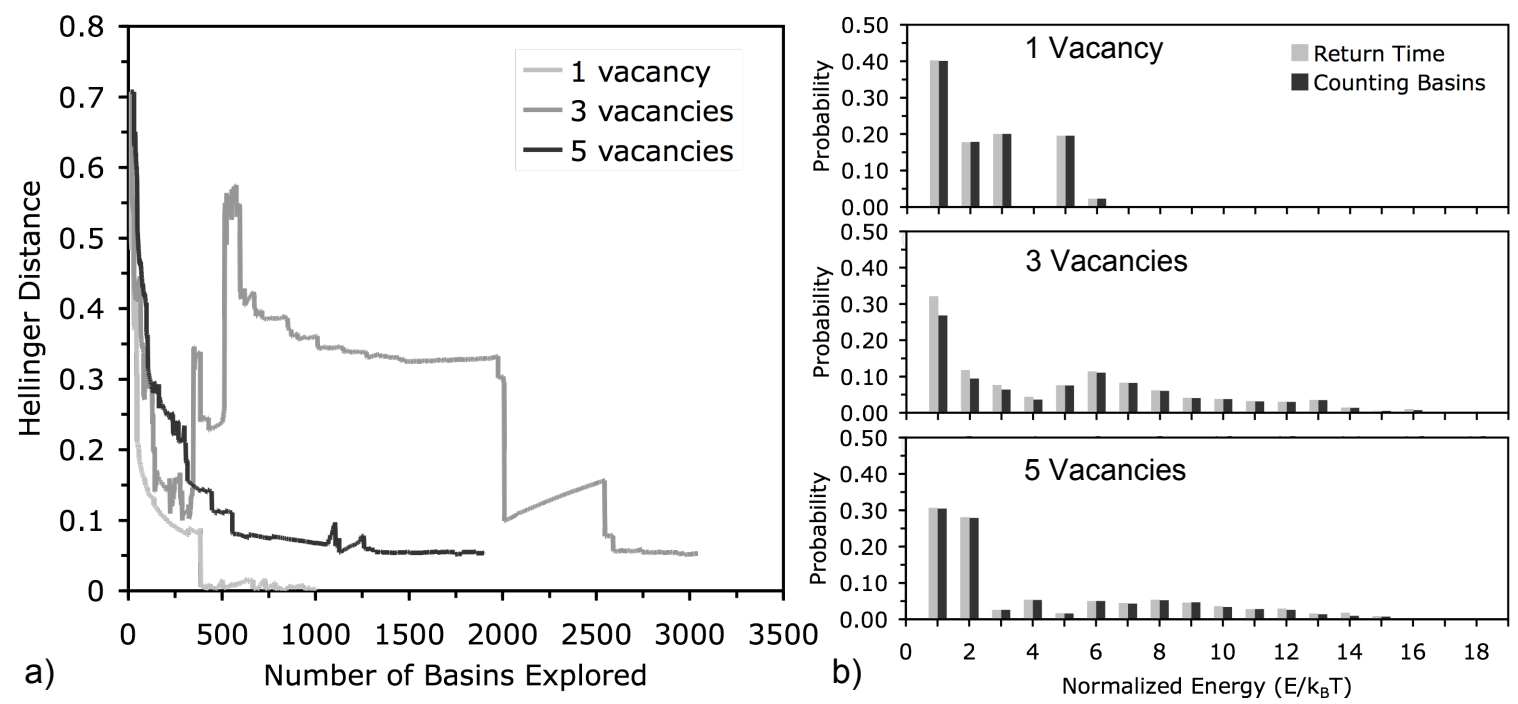

Figure 3: a) Hellinger distance between the two distributions $p^{\text {count }}$ and $p^{\text {return }}$ used here, versus the number of energy basins explored, for three different AMD simulations containing an edge dislocation (as in Figure 2d) and $n=1,3$ and 5 vacancies, respectively. Using three convergence criteria (see text), the controlling criterion for convergence (1000 basins explored; Hellinger distance $<0.1$ for 500 basins or more; average number of basins explored per occupied energy bin $>100)$ was different for each of these simulations $(n=1: 1000$ basins explored; $n=3$ vacancies: Hellinger distance; $n=5$ vacancies: number of basins explored per occupied energy bin). b) The two distributions $p^{\text {count }}$ and $p^{\text {return }}$ at convergence, for all cases $n=1,3,5$.

Once the AMD attains near-equilibrium, we use the resulting information on the distribution of point defects in Zones 2 and 3 to establish boundary conditions for the subsequent Discrete Diffusion simulation. First, the total number of point defects contained in Zone 2 or 3 , where point defects are well-defined within the distorted crystalline lattice, is computed as the fraction of time any point defects spends in Zone $z=2$ or $z=3$ during the accelerated MD simulation

$$
N_{z}^{M D}=\frac{\sum_{j=j_{0}}^{j_{f}} m_{j}^{(z)} t_{j}}{\sum_{j=j_{0}}^{j_{f}} t_{j}},
$$

where $m_{j}^{(z)}$ was defined earlier as the total number of point defects in Zone $z$ in the $j^{\text {th }}$ energy basin. Recall that Zone 2 was included in the dynamics only so that the the sampling of point defect concentrations in Zone 3 is not affected by the fixed atom boundary conditions in Zones 0 and 1. Since the $n$ defects that are inserted into the AMD simulation have already passed from Zone 2 into Zone 3 by the diffusion equation, the total number of point defects measured in Zone 2 must be redistributed back into Zones 3 and 4 so that all $n$ defects are contained within Zones 3 and 4. The fraction of $N_{2}^{M D}$ that is distributed back into Zone 3 is given by

$$
\Delta N_{3}^{M D}=N_{2}^{M D} \frac{N_{3}^{M D}}{n-N_{2}^{M D}}
$$

The total number of point defects in Zone 3 is then $N_{3}^{M D}+\Delta N_{3}^{M D}$. These defects are then distributed among all the sites $i$ in Zone 3 according to the concentrations computed using the site 
This is a pre-print of the following article: Baker, K. L.; Curtin, W. A. J. Mech. Phys. Solids 2016, 92, 297-312.. The formal publication is available at http://dx.doi.org/10.1016/j.jmps.2016.04.006

formation enthalpies (Eq. 5) as

$$
c_{i}^{(3, M D, n)}=\frac{e^{-\beta \Delta H_{i}^{v}}}{\sum_{i \in 3} e^{-\beta \Delta H_{i}^{v}}}\left(N_{3}^{M D}+\Delta N_{3}^{M D}\right),
$$

where $\beta=1 / k_{B} T$. This computation of the site concentrations in Zone 3 is performed because it is too computationally intensive to allow AMD to explore all possible positions of all the point defects to achieve a full equilibrium of point defects in Zone 3. Therefore when near-equilibrium is reached in an AMD simulation, the individual site concentrations will not precisely match the true equilibrium distribution. However, the total averaged number of point defects measured in Zones 2 and 3 (i.e. the sum of the site concentrations) converges much faster. Thus, above, we compute the total number of point defects in Zone 3 and analytically compute the individual equilibrium site concentrations in Zone 3. The computed site concentrations in Zone 3 in the presence of $n$ and $n+1$ defects in Zones 3 and 4, i.e. $c_{i}^{(3, M D, n)}$ and $c_{i}^{(3, M D, n+1)}$, are then used to updated by the Discrete Diffusion equation at any time in the interval $t_{n}<t<t_{n+1}$ as described in the previous section.

\section{Application: Edge Dislocation Climb in Aluminum}

As a demonstration, the DD-MD algorithm is applied to study the transport of vacancy defects into an edge dislocation, which is associated with the nucleation and expansion of jog pairs that represents the initial stages of dislocation climb. Because the dislocation is an extended defect, we can only study evolution of a short segment of an infinite line, without addressing issues such as pipe-diffusion along the dislocation core over long distances. Thus, this preliminary application is not a full study of the problem of dislocation climb. There is a long-standing theoretical framework for double-jog nucleation $[16,17,18,19,20]$ but, because of the huge range of spatial and temporal scales involved, only a few recent attempts have been made to model the climb process at the atomistic scale $[21,22,23,24]$. We discuss our simulations with respect to theory and earlier models in the discussions sections.

\subsection{Geometry and Material Properties}

We simulate an edge dislocation core in Aluminum at $T=600 \mathrm{~K}$. The crystal orientation has

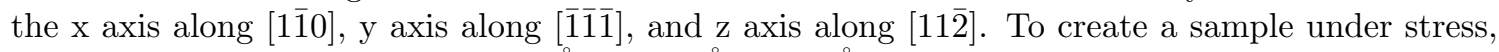
a fully-periodic crystal of size $308.0 \AA \times 307.3 \AA \times 44.4 \AA$ is equilibrated under the desired uniform applied stress tensor (specified below). An edge dislocation is then created in a two-step procedure. First, an edge dislocation is created by superimposing the elastic displacement field for a perfect edge dislocation displacement with the Burgers vector $\mathbf{b}=a / 2[1 \overline{1} 0]$ (where $a$ is the lattice constant of $4.032 \AA$ ) and line direction along the $\mathrm{z}$ axis. Atoms within $10 \AA$ of the outer boundary along $\mathrm{x}$ and $\mathrm{y}$ were then held fixed and the remaining atoms then relaxed to a minimum energy structure using molecular statics in LAMMPS [25] to create the relaxed dislocation core structure, which consists of two partials separated by a stacking fault. To reduce the computational cost of subsequent simulations, a much smaller cylindrical sample with radius $70 \AA$ around the dislocation line is extracted and the outer $10 \AA$ of this smaller cylinder are then held fixed during the simulation. We define Zones 0-4, which contain 13815, 26352, 612, 504, and 684 atoms, respectively, with the final configuration shown in Figure 2. The fraction of Zone 3+4 atoms within Zone 3 is thus $\sim 0.42$. 
The Ercolessi and Adams embedded atom potential for Aluminum is used [26]. For this potential, the vacancy formation enthalpy at zero pressure and temperature is $\Delta H^{f}=0.69 \mathrm{eV}$ and the vacancy misfit volume is $\Delta V^{f}=9.75 \AA^{3}[12]$. At $T=600 K$, the vacancy concentration in bulk $\mathrm{Al}$ is then $c=1.6 \times 10^{-6}$. The vacancy migration enthalpy at zero temperature and pressure is $\Delta H^{m}=0.61$ $\mathrm{eV}$ and the principal values of the vacancy misfit volume tensor at the transition state for site-tosite hopping are $(11.15,-1.97,-5.74) \AA^{3}$, where the first value corresponds to the $\langle 100\rangle$ direction perpendicular to jump direction, the second value corresponds to the $\langle 110\rangle$ jump direction, and the third value corresponds to the $\langle 110\rangle$ direction perpendicular to jump direction [12].

In the discrete diffusion simulations, we use an attempt frequency $\nu_{0}=1 \times 10^{13} / \mathrm{s}$. For the bond-boost hyperdynamics method, we use a value of 0.35 for the maximum bond strain $\left(\epsilon_{\alpha}\right.$ from [2]) and a maximum boost-energy $\left(S_{\alpha}\right.$ from [2]) that is determined adaptively as the simulation proceeds to maintain efficient sampling of the boosted potential yet provide accelerated transitions to configurations that would sampled less frequently using standard MD. Appendix A provides an overview of the self-learning bond-boost hyperdynamics procedure, with further details provided in $[2]$.

The applied stress tensor is chosen such that there is a large climb force on the dislocation but with a pressure near zero. The far-field vacancy concentration is thus independent of the applied stress field. This is achieved by applying a displacement field that generates a compressive uniaxial stress in the Burgers vector direction with corresponding tensile stresses in the perpendicular directions to keep the pressure at zero,

$$
\boldsymbol{\sigma}=\left[\begin{array}{ccc}
\sigma_{x x} & 0 & 0 \\
0 & -\sigma_{x x} / 2 & 0 \\
0 & 0 & -\sigma_{x x} / 2
\end{array}\right] \mathrm{MPa},
$$

with $\sigma_{x x}<0$. Since $\mathrm{Al}$ is slightly anisotropic and the elastic constants depend on temperature, zero pressure is not achieved exactly, but the differences are negligible. Here, we consider an applied stress of $\sigma_{x x}=-972.1 \mathrm{MPa}$ with a small residual pressure $p=-13.7 \mathrm{MPa}$ and shear $\sigma_{y z}=65.5$ MPa.

We envision a region of bulk material at the equilibrium vacancy concentration $c$ into which a dislocation glides and arrests at the origin at time $t=0$. Thus, the initial concentrations in all Zones are set to $c$. However, the vacancy concentration boundary condition in Zone 0 is then set to the eventual concentration in the presence of the dislocation. This ensures that the system (simulation cell) eventually reaches a correct equilibrium with the bulk concentration of vacancies, but also causes a (small) artificial jump in the initial site concentrations between Zones 0 and 1 (maximum difference of $28 \%$ ). At the equilibrium concentration, and with $\sim 1000$ atoms in Zones $3+4$, there are only a total of 0.002 vacancies in these regions at $t=0$. The case of 0 vacancies requires no AMD, and so the first AMD simulation contains 1 vacancy in Zones 3+4.

\subsection{Results: Equilibrium}

The AMD simulation with one vacancy in Zones $3+4$ predicts that there are 0.20 vacancies in Zone 3. If the one vacancy were simply equally distributed among all sites in Zones 3 and 4, the concentration in Zone 3 would be $\sim 0.42$. Thus, the lower measured value of 0.20 indicates that there is weak binding of the single vacancy around the dislocation. The Discrete Diffusion algorithm is then executed for Zones 0-3 using boundary conditions interpolated from the AMD concentrations for Zone 3 for 0 and 1 vacancy, as described earlier. The predicted total number of vacancies in Zones 3+4 versus time is shown in Figure 4a. Equilibrium is reached within a few 
This is a pre-print of the following article: Baker, K. L.; Curtin, W. A. J. Mech. Phys. Solids 2016, 92, 297-312.. The formal publication is available at http://dx.doi.org/10.1016/j.jmps.2016.04.006

microseconds, at which point the net flux into Zone 3 becomes zero. At equilibrium, the total number of vacancies in Zone 3 is $1.36 \times 10^{-3}$ and in Zones $3+4$ is $6.89 \times 10^{-3}$. With far less than one vacancy in the dislocation core region, there is no nucleation of any jog structure and no climb, in spite of the very large applied climb force.

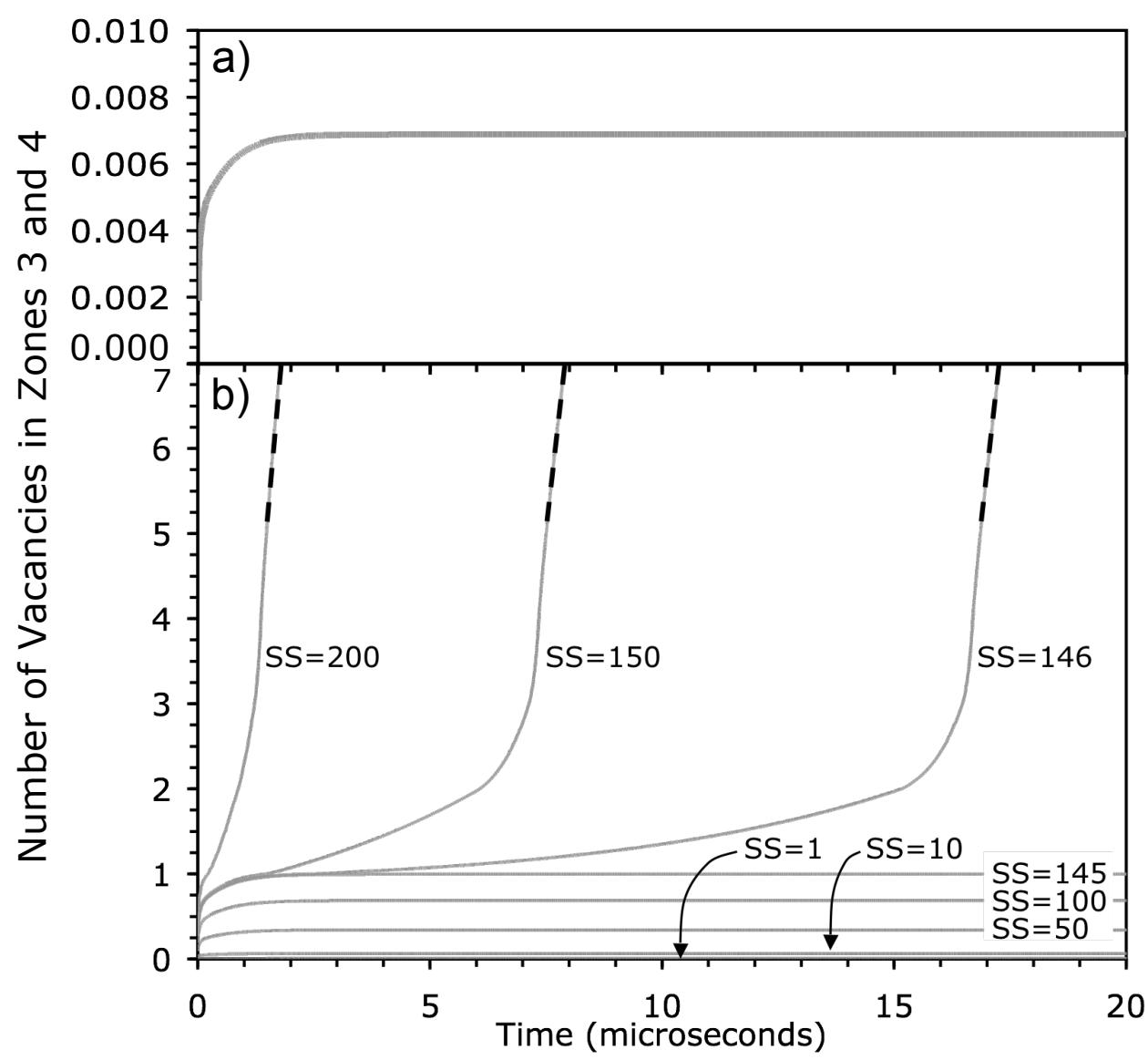

Figure 4: a) Number of vacancies in Zones $3+4$ versus time, for an edge dislocation initially placed into the bulk crystal. Equilibrium is achieved with far less than one vacancy in Zones $3+4$ so that no climb processes occur. b) Number of vacancies in Zones $3+4$ versus time with labels indicating the imposed vacancy supersaturation in the bulk crystal. Below a supersaturation of 146 , the total number of vacancies plateaus to an equilibrium concentration. For a supersaturation of 146 or greater, the number of vacancies in Zones $3+4$ increases continuously, due to the nucleation of a double-jog that acts as a vacancy sink. The dotted lines indicate a constant, steady-state rate of vacancy absorption after the double-jog becomes a sink for vacancies.

The total number of vacancies is proportional to the length of the simulation cell (equal to the dislocation line length, $44.44 \AA$ ). To achieve two separated vacancies inside the atomistic region would require a simulation cell/dislocation line of $12905 \AA=2(44.44 \AA) / 6.89 \times 10^{-3}$. The two vacancies could then possibly merge to form a different more-stable defect such as a tiny jog. For an even longer simulation cell, the total number of vacancies could increase further. However, the 
This is a pre-print of the following article: Baker, K. L.; Curtin, W. A. J. Mech. Phys. Solids 2016, 92, 297-312.. The formal publication is available at http://dx.doi.org/10.1016/j.jmps.2016.04.006

binding energy of two or more vacancies (including any work done by the applied stress) would need to be sufficiently large to overcome the entropy associated with the localization of the vacancies. The energies of defects with additional vacancies are shown further below, and would form the basis for estimates of their formation under equilibrium conditions, but such an investigation is outside the scope of the present study.

\subsection{Results: Climb with a Vacancy Supersaturation}

To drive jog nucleation in the (small) atomistic region requires the imposition of non-equilibrium conditions. Since the equilibrium number of vacancies in Zone 3 is 145.2 times smaller than the prediction from AMD with one vacancy, the supersaturation must be greater than 145.2 to absorb the first vacancy. We thus run the same dynamic simulation but with far-field vacancy supersaturations of $10 \times, 50 \times, 100 \times, 145 \times, 146 \times, 150 \times$, and $200 \times$ the equilibrium far-field concentration. The number of vacancies in Zones $3+4$ versus time for each vacancy supersaturation is shown in Figure 4b. For supersaturations of 145 or lower, the system reaches equilibrium prior to the injection of a single vacancy into Zones $3+4$. For a supersaturation greater than 145 , the system does not reach equilibrium in Zones 3+4 and, after a transient period of 1-15 microseconds, Zones 3+4 steadily absorb vacancies that flow in from the surrounding region. In this regime, the predicted number of vacancies in Zone 3 as a function of the total number of vacancies in Zones $3+4$ evolves as shown in Figure 5. At these supersaturations values, when more than one vacancy is introduced into Zones $3+4$, the vacancies bind together near the core and become less likely to appear in Zone 3 . The concentration in Zone 3 thus remains below the "equilibrium" value (sum of the site concentrations $c=e^{-\beta \Delta H^{v}}$ multiplied by the supersaturation) and far-field diffusion thus injects additional vacancies into Zones $3+4$ that move into Zone 4 . When 2 or 3 vacancies exist in Zones $3+4$, their binding is moderate (see below) so that the decrease in concentration in Zone 3 is small. But since it remains below the equilibrium value, further vacancies diffuse in from Zones 0-2. When 4 vacancies have diffused into Zones 3+4, the AMD-measured number of vacancies in Zone 3 becomes almost zero, indicating strong binding of the 4 vacancies to the dislocation to create some sort of very stable defect (see below). When 5 vacancies have diffused into Zones $3+4$, the measured number of vacancies in Zone 3 becomes zero to within the limits of computational accuracy; this indicates that a vacancy sink has formed in the defect region (see below). Additional vacancies flow from the far-field into Zone 4 and are absorbed at the sink and thus never diffuse out into Zone 3 , independent of the supersaturation above the critical value. The coupled simulations are halted before the $8^{\text {th }}$ vacancy is absorbed. 
This is a pre-print of the following article: Baker, K. L.; Curtin, W. A. J. Mech. Phys. Solids 2016, 92, 297-312.. The formal publication is available at http://dx.doi.org/10.1016/j.jmps.2016.04.006

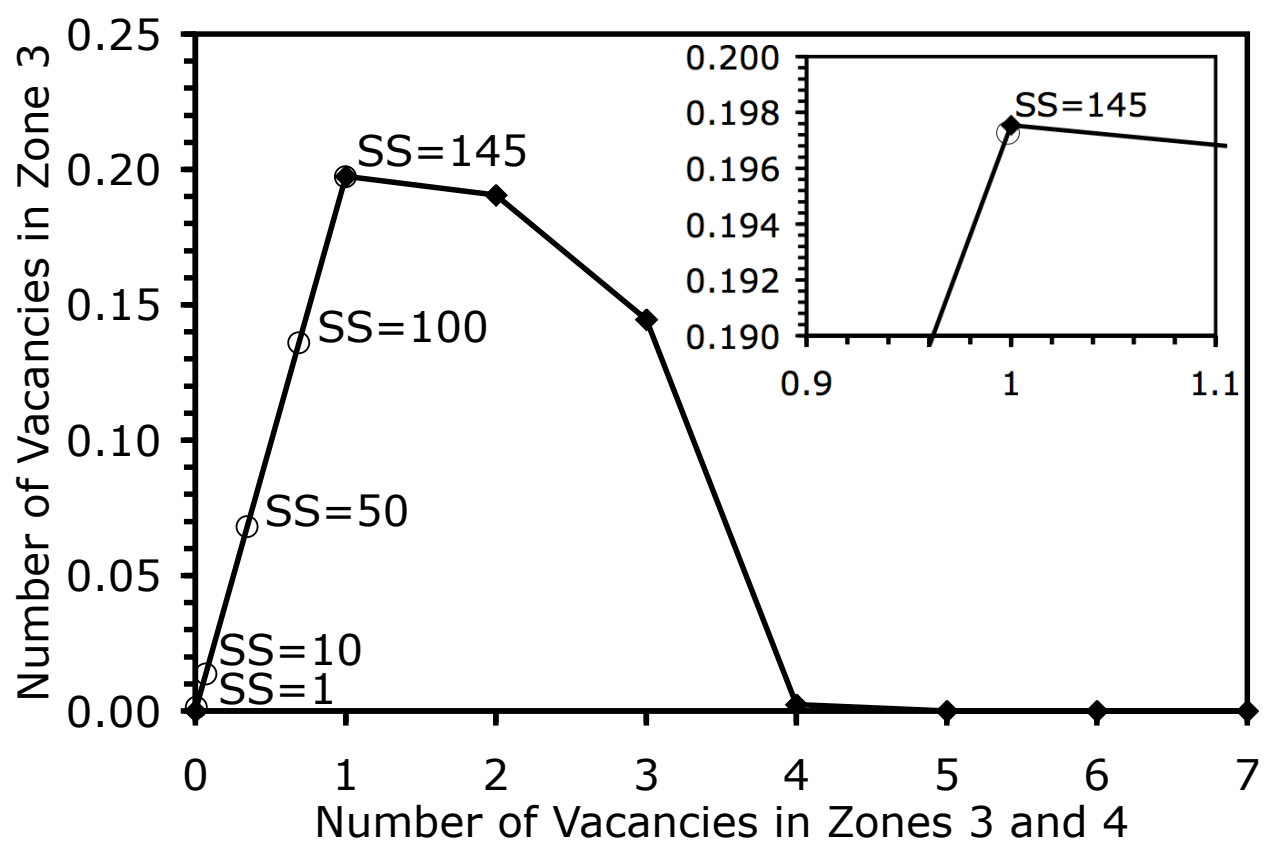

Figure 5: Number of vacancies in Zone 3 versus the number of vacancies that have flowed into Zones 3 and 4 . Solid symbols are computed and the lines are a guide to the eye. The open symbols, labeled by the imposed vacancy supersaturation, show the equilibrium number of vacancies in Zone 3 achieved for supersaturations below 146. For supersaturations of 146 or larger, the "equilibrium value", calculated by summing the site concentrations using the formation enthalpies in Zone 3 multiplied by the bulk concentration, cannot be obtained because the vacancies remain trapped near the dislocation. The inset figure provides a close up around the values corresponding to 1 vacancy in Zones 3 and 4.

The creation of a sink is confirmed by measuring the potential energy change in the atomistic domain upon adding one additional vacancy into the AMD simulation, $\Delta E(n)=\left(E(n)+E_{a}\right)-$ $E(n-1)$, where $n$ is the number of vacancies and $E_{a}$ is the potential energy of an atom in the bulk under zero stress, $-3.36 \mathrm{eV}$ [26]. The inclusion of $E_{a}$ accounts for the potential energy of the atom that is replaced by the new vacancy after it has moved far away from the dislocation core out of the AMD domain. The addition of a new vacancy into the AMD domain has two contributions: the bulk vacancy formation energy $\Delta H^{f}=0.69 \mathrm{eV}$ and the binding energy $\Delta E^{b}(n)$ due to interactions of the $n^{t h}$ vacancy and the defect including the previous $n-1$ vacancies, $\Delta E(n)=\Delta E^{b}(n)+\Delta H^{f}$. If the cost to add a vacancy is greater than zero, $\Delta E(n) \geq 0$, then equilibrium between the far-field Zones 0-2 and the defect structure in Zones $3+4$ can be reached. In this regime, the defect acts as a trap that absorbs and emits vacancies. When the cost of adding a vacancy is negative, $\Delta E(n)<0$, the system gains energy by adding a vacancy to the defect, and a sink exists. A sink annihilates the vacancy, lowering the energy by more than the vacancy formation energy $\Delta H^{f}$ relative to a vacancy far from Zone 4. In the presence of the sink, there is a driving force for the flow of vacancies from the bulk crystal (which is in equilibrium with a surface far away from the simulation domain) into the defect structure. Figure 6 shows the incremental potential energy cost to add a vacancy $\Delta E(n)=\left(E(n)+E_{a}\right)-E(n-1)$ where $E(n)$ and $E(n-1)$ are taken as the potential energies of the minimum energy structures discovered in the respective AMD simulations. When 1 vacancy enters 
This is a pre-print of the following article: Baker, K. L.; Curtin, W. A. J. Mech. Phys. Solids 2016, 92, 297-312.. The formal publication is available at http://dx.doi.org/10.1016/j.jmps.2016.04.006

Zones $3+4$, the energy cost is only $0.18 \mathrm{eV}$ lower than the vacancy formation enthalpy, indicating weak binding to the dislocation. When 2,3 , and 4 vacancies are in Zones $3+4$, the binding energies are all positive but $\sim 0.4-0.5 \mathrm{eV}$ below the formation enthalpy, indicating stronger binding and less probability of finding vacancies in Zone 3 . When the $5^{\text {th }}$ vacancy diffuses into Zone 4 , the cost to adding a vacancy decreases precipitously, and becomes negative. Moreover, the energy gain is nearly equal to the work done by the climb force on the dislocation, $\sigma_{x x} v_{a}=0.099 \mathrm{eV}$ where $v_{a}$ is the volume per atom. Additional vacancies $(6,7, \ldots)$ diffuse into Zones $3+4$ and are absorbed into the sink, with an energy change corresponding to the work done by the applied stress. Once the sink is formed, AMD is unable to measure a finite vacancy concentration in Zone 3 within reasonable times because the energy cost of removing a vacancy from the sink to Zone 3 is large, approaching $\Delta H^{f}+\sigma_{x x} v_{a}$. We note that in these simulations, the AMD simulations would probably measure zero concentration in Zone 3 even at zero stress, since the jog annihilates the vacancy and requires approximately $0.69 \mathrm{eV}$ to release it again. Thus, the simulations here that measure zero concentration in Zone 3 do not correspond precisely to the true situation for double-jog nucleation and growth; this difficulty is address more fully in the discussion section.

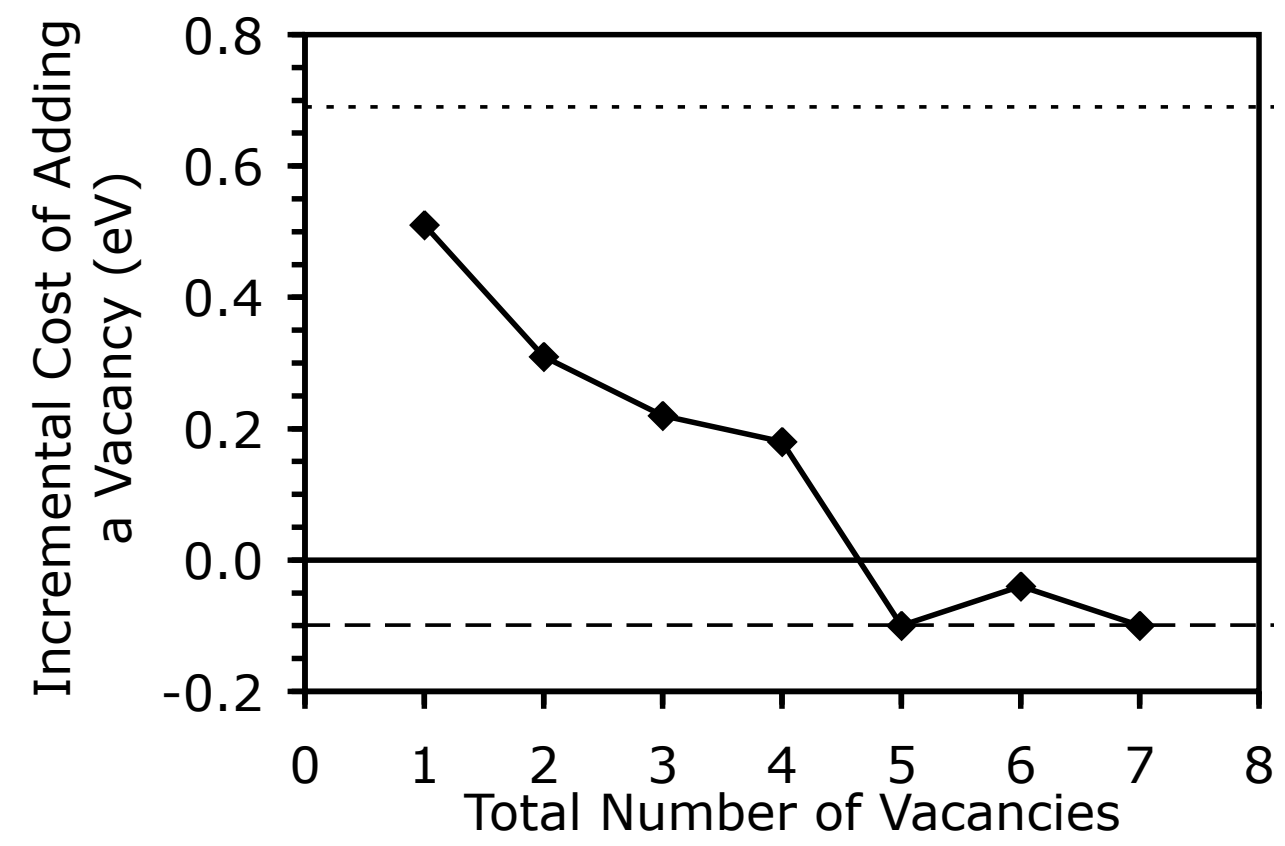

Figure 6: A plot of the change in the total potential energy of the system between each AMD simulation. When the energy change is negative, the system gains energy by placing a vacancy into the defect indicating that a sink has been formed. The dotted line corresponds to the vacancy formation enthalpy in the bulk, and the dashed line corresponds to $-\sigma_{x x} v_{a}$.

The various atomic structures associated with double-jog nucleation due to vacancy absorption have not been examined previously using molecular dynamics, and so we show some results emerging from our extensive AMD simulations. Figure 7 shows some of the low-energy configurations that dominate the AMD for various numbers of vacancies introduced into Zones 3+4. As was surmised 
This is a pre-print of the following article: Baker, K. L.; Curtin, W. A. J. Mech. Phys. Solids 2016, 92, 297-312.. The formal publication is available at http://dx.doi.org/10.1016/j.jmps.2016.04.006

through inspection of the energies, when 3 vacancies are present, they coalesce into a defect that reside near one of the partials of the dissociated core. This defect dissociates and reforms over time. When 4 vacancies are present, they form a prismatic dislocation loop on one of the partial dislocation cores, as seen in more detail in Figure 8 and as postulated by Hirsch [27]. This loop can diffuse between the two partial dislocation cores or form a double-jog structure corresponding to dislocation to climb. The 4-vacancy dislocation loop very occasionally decomposes by emitting a vacancy that diffuses into Zone 3 , but in general the loop acts as a strong trap for vacancies in the core region. Once 5 or more vacancies are absorbed, a distinct double jog is created that rarely, if ever, decomposes into a smaller dislocation loop/jog structures with separated vacancies. The double jog then acts as a perfect sink: vacancies incorporated into the jog never detach from the jog within time scales measurable in AMD.

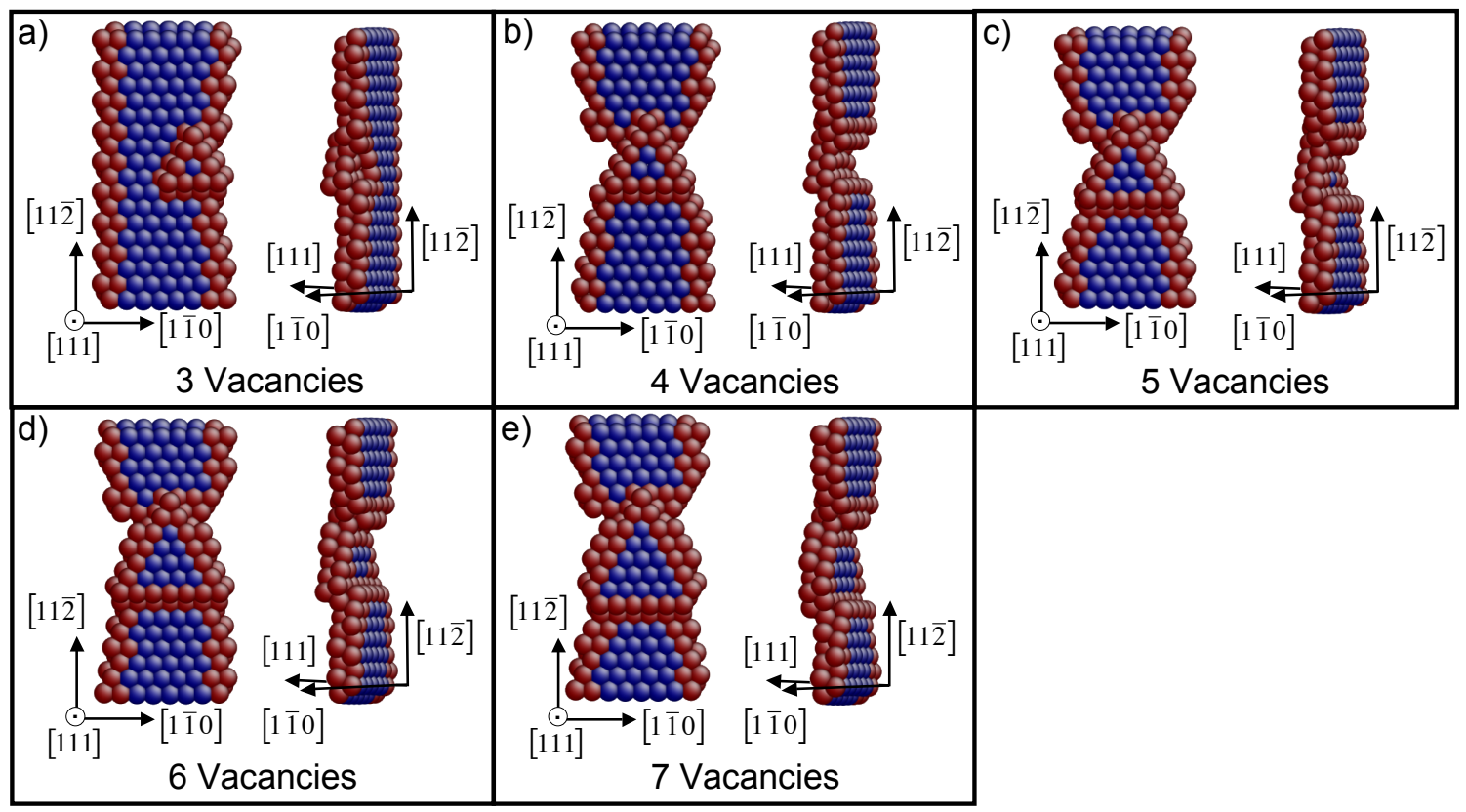

Figure 7: (Color online) The atomic structure of typical dislocation structures when a) 3, b) 4, c) 5, d) 6, and e) 7 vacancies are present in the dislocation core. The color corresponds to the CNA of each atom, where the red atoms are disordered and the blue atoms have HCP stacking. Atoms with FCC stacking are not shown. Each structure is shown looking down on the dislocation slip plane and from the sides so that the 3D structure is visible. 
This is a pre-print of the following article: Baker, K. L.; Curtin, W. A. J. Mech. Phys. Solids 2016, 92, 297-312.. The formal publication is available at http://dx.doi.org/10.1016/j.jmps.2016.04.006

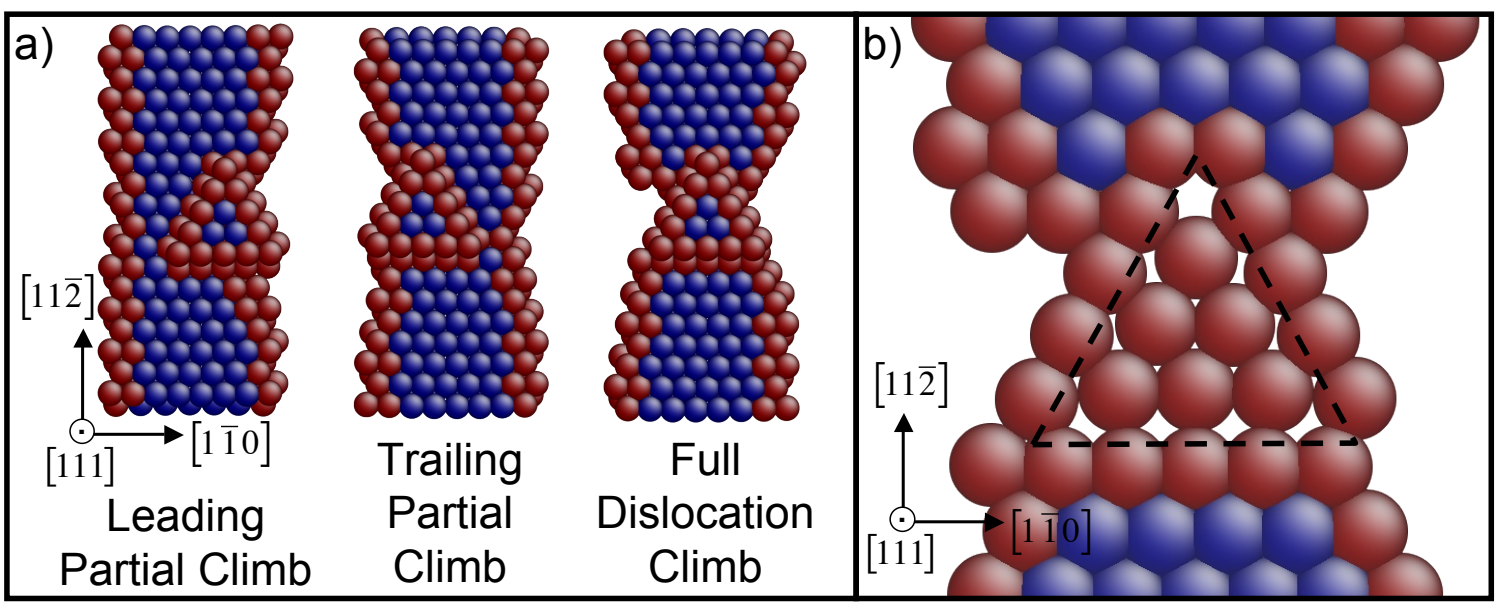

Figure 8: (Color online) a) The atomic structure of three typical prismatic loop and double jog configurations when four vacancies are present in the AMD simulation. The color corresponds to the CNA of each atom, where the red atoms are disordered and the blue atoms have HCP stacking. Atoms with FCC stacking are not shown. b) A close-up of the full dislocation double-jog, only showing the plane of atoms on the top of the original slip plane (with the jogged plane of atoms removed), in which the dislocation structure closely resembles a partial-vacancy structure described by Hirsch [27].

Once the sink has formed, i.e. after 5 vacancies have been absorbed, the number of vacancies in Zone 3 remains zero and the system approaches a steady state with a near-constant flux of vacancies into the dislocation, as indicated by the dashed lines in Figure 4. We have measured the flux at the point where the simulations have been halted (not perfect steady state), and it is found to be linearly proportional to the applied background concentration. This behavior is expected because analysis of the diffusion equation in the presence of a sink shows that the flux into the sink is proportional to the far-field vacancy concentration, and our coupled DD-MD algorithm faithfully reproduces the full diffusion problem.

\subsection{Discussion on Simulations of Dislocation Climb}

Our results show that, starting from a straight dislocation, double-jog nucleation does not occur even with a large climb force $\left(\sigma_{x x}=0.97 \mathrm{GPa}\right)$. This may seem at odds with standard theory for climb. However, in the absence of jogs, the climb force plays no role since it only does work on the system when an existing jog moves by absorption of a vacancy; our result is thus physical within the context of the simulation conditions. Standard climb theory (e.g. as in Hirth and Lothe [16]) implicitly assumes the existence of a jog, even if the diffusion problem is solved for an infinite straight jog-free dislocation. In the theory, the existence of the jog is indirect, via imposition of an effective vacancy concentration near the core that is enhanced by the work done by the climb force. Thus, theory and simulation on nominally the same problem are not actually studying the same microscopic problem.

Atomistic simulations can only study a short segment of dislocation. For an infinite dislocation, equilibrium corresponds to a finite (although very small) density of double-jogs of various lengths along the core, as dictated by the formation energy of such jogs [16, 28]. Therefore, simulations of climb perhaps should probably start with an initial double jog of some length, focusing on the (rare) 
atomistic region where such a double jog has formed at equilibrium. However, such simulations will then most likely predict that double jog lengthening (climb) will occur at zero driving force because the double jog acts as a sink (the jog never emits a vacancy). Since the introduction of a vacancy "by hand" into the MD region automatically increases the potential energy of the MD system by approximately the vacancy formation energy $\Delta H^{f}$, the absorption of the vacancy into the sink will then occur naturally in the MD since this process lowers the system energy by $\sim \Delta H^{f}$ even at zero climb force. Missing in such an MD simulation is the complementary behavior occurring elsewhere in the real material where vacancies are actually injected into the bulk (e.g. near free surfaces). It is in these regions that the system energy is first increased by $\sim \Delta H^{f}$ upon injection of vacancies. In equilibrium under zero climb force, this energy is exactly regained/balanced by vacancy absorption into the dislocation jog sink. Conversely, a dislocation jog can emit a vacancy back into the lattice, at a cost of $\Delta H^{f}$, which is regained/balanced by absorption of a vacancy at the surface. The nucleation process that creates the necessary vacancies in the bulk, at a cost of $\Delta H^{f}$, is not easily achieved within atomistic simulations. MD simulations of climb must thus be supplemented by global energetic considerations to provide meaningful information on climb rates, although the MD simulations do provide information about the local processes/configurations around the jogs.

Dislocation climb is complex and rich, which is why the details of the climb process are still poorly understood. Here, using a rigorous AMD approach within the coupled simulation, detailed atomistic processes and structures are revealed that provide a wealth of new information about the jog nucleation and climb process. Future work will provide a more-detailed analysis of the thermodynamics, kinetics, and atomistic processes associated with the climb process.

\section{Discussion and Summary}

The coupled DD-MD method presented here provides a path to studying some challenging problems involving point defect diffusion into extended defects. However, there are some important limitations that merit discussion.

First, as evident in our discussion of dislocation climb, even AMD is not capable of efficiently simulating local equilibrium point defect concentrations in systems with strong traps or sinks. For problems with complex defects, identifying the emergence of a sink is not simple. One signature of a sink, as opposed to a trap, is a reduction in the potential energy in the MD domain equal to or larger than the bulk point defect formation energy (as in Figure 6). Another associated signature is the measurement of essentially zero point defect concentrations in Zone 3 (as in Figure 5). Once a sink is formed, local AMD simulations (with or without coupling to a far field) will usually miss the global energetics associated with point defect nucleation far from the sink.

Second, this DD-MD method provides a deterministic, equilibrium approach to point defect diffusion into the distributed defect. Fluctuations in the point defect concentration field that occur in real materials may cause fundamentally different behavior from the equilibrium diffusion solution. In order to account for fluctuations, a stochastic model would be needed. A stochastic model might require pre-knowledge of attainable fluctuations and might require use of stochastic boundary conditions in coupling to the AMD measurements (which must also measure or quantify the fluctuations in the number of vacancies measured in Zone 3).

Third, in substitutional solid solutions, the solute atom diffusion is meditated by vacancies. This can be handled at the Discrete Diffusion level by introducing coupled equations for the evolution of both vacancies and solute atoms. However, vacancy concentrations in metals are quite low so that a single vacancy may never be injected into the AMD domain. And, even if one vacancy is artificially 
added to the defect to facilitate solute diffusion, the diffusion of the solute atoms only occurs during a small fraction of the vacancy hops and so AMD must be run for much longer times, increasing the computational demand. Depending on the distributed defect, using an on-the-fly kinetic Monte Carlo approach may be more efficient once many of the migration barriers are discovered. As mentioned above, fluctuations in the vacancy concentration may also be important to this class of problems since a small increase in the number of vacancies in the defect may lead to very different defect structures or rates of equilibration.

Fourth, the linear interpolation of the Zone 3 point defect site concentrations between the values calculated using AMD is an approximation that could be enhanced.

Fifth, AMD is computationally expensive since it involves waiting for transition events to occur, even if they are at an accelerated rate. Fortunately, different types of AMD can be combined to simulate longer MD times. Hyperdynamics, used here, can be combined with the parallel replica method [6]. In this approach, many independent instances of the same AMD system are executed using hyperdynamics until one system transitions to a new state, at which point all systems are updated to the new state and all replicas then evolve further from that point to create a single dynamic trajectory at an accelerated pace. Also, since the objective of the AMD simulations is to measure the average number of point defects in a specific region, parallel sampling of phase space can be done using many independent hyperdynamics simulations run in parallel. Since each AMD simulation uses a relatively small number of atoms, multiple dynamic trajectories could be run independently on a large number of processors and the trajectories combined to measure averages more accurately.

In spite of these limitations, the present method has merits compared to other more computationally efficient methods such as the phase-field-crystal method [29] and diffusive molecular dynamics [30] that have been recently introduced to study similar classes of problems. The currently-implemented PFC methods addressing the evolution of distributed defects from point defect diffusion $[21,31,32]$ lack an accurate description of the true atomic-scale point defects, with neither defect structures, energies, nor transition rates, computed accurately [33]. Related to the application here, while "climb" processes have been simulated, the nucleation processes are not captured with true atomistic fidelity and climb mechanisms that do not rely on double kink nucleation are reported [21]. The DMD method combines a classical density functional approach for the free energy with a Master Equation-type diffusion model to follow the evolution of defects over "diffusive" time scales [24,30]. The energetics of any atomic state are thus accurately described in DMD but the time evolution remains approximate because the method does not use actual atomic transition barriers in the region around the complex evolving defect. In problems/regions with small point defect concentrations and concentration gradients, there is no need for atomistic detail and so, while all three methods become similar, the Discrete Diffusion algorithm here is by far the most efficient. All of the methods have similar difficulties with the simulation of sinks, which is a general issue of molecular-level simulation methods. Also, none of the methods incorporate natural fluctuations in the point defect concentration without adding them artificially. Since PFC and DMD are far more computationally-efficient than the current method, the choice of method depends on the level of realism and quantitative accuracy being sought in the simulation.

In summary, we have presented a concurrent multiscale approach to model transient mass diffusion over long spatial and temporal scales using detailed atomistic modeling to capture evolution of complex defects as point defects are absorbed or emitted into/from the defect. The coupled DD-MD algorithm is applicable to a general atomistic system with interstitial or substitutional point defects. Important algorithmic details have been presented, including the key issue of coupling between the 
discrete-diffusion and fully-atomistic domains and also the assessment of near-equilibrium during the fully-atomistic AMD simulations. The latter issue transcends the coupled method and may find value in assessment of convergence in other AMD studies. The coupling DD-MD methodology has been demonstrated using the problem of vacancy diffusion and absorption into an edge dislocation core in Aluminum to cause dislocation climb, with various insights into the climb process revealed. Beyond the current application, the new DD-MD algorithm represents a powerful method to study many physical problems where the transient evolution of crystalline defects is controlled by longrange diffusion of point defects from the surrounding crystal. Taking advantage of both accelerated atomistic simulations and a rate theory approach, defect structures and bulk diffusion are both captured accurately in this new method.

\section{Acknowledgements}

All atomic structures were visualized using AtomEye [34]. Support for this work was provided through a European Research Council Advanced Grant, "Predictive Computational Metallurgy", ERC Grant agreement No. 339081 - PreCoMet.

\section{Appendix A: Self-Learning Bond-Boost Hyperdynamics}

Our implementation of self-learning bond-boost hyperdynamics is based on the work of Perez et al. 2009 [2]. They state that the potential of the mean force (PMF) for each pair of nearest neighbor atoms defining a bond $i$ can be estimated as a function of the strain in bond $i, \epsilon_{i}$, as

$$
\operatorname{PMF}_{i}\left(\epsilon_{i}\right)=-k_{B} T \ln \left[\frac{\left\langle\rho_{i}\left(\epsilon_{i}\right)\right\rangle}{\left\langle\rho_{i}\left(\epsilon_{i}=0\right)\right\rangle}\right]
$$

where $\left\langle\rho_{i}\left(\epsilon_{i}\right)\right\rangle$ is the ensemble average of $\rho_{i}\left(\epsilon_{i}\right)$, which is the probability of finding bond $i$ at a bond strain strain of $\epsilon_{i}$. The bond strain of any bond $i$ is given by

$$
\epsilon_{i}=\frac{r_{i}-r_{i}^{e q}}{r_{i}^{e q}}
$$

where $r_{i}$ is the instantaneous distance between the two atoms that make up bond $i$, and $r_{i}^{e q}$ is the equilibrium distance between the atoms of bond $i$.

In this work, we use time averages to approximate the ensemble averages. The average probability of finding a bond with a bond strain of zero must be computed with standard MD, and is computed by

$$
\left\langle\rho\left(\epsilon_{i}=0\right)\right\rangle=\frac{1}{t_{t o t}^{M D}} \sum_{\substack{j=1 \\ \epsilon_{i} \in[0, q / 20)}}^{N \text { steps }^{M D}} \Delta t,
$$

where $t_{t o t}^{M D}$ is $5 \mathrm{ps}$, so that $N$ steps ${ }^{M D}=t_{t o t}^{M D} / \Delta t=5000$, and $\Delta t$ is the time step of $1 \mathrm{fs}$. This value is computed prior to turning on the bond-boost potential, and is observed to converge rapidly within the 5 ps sampling time. After standard MD is run for $5 \mathrm{ps}$, the bond-boost potential is turned on and ensemble average at $\epsilon_{i}=q$ is computed by

$$
\left\langle\rho\left(\epsilon_{i}=q\right)\right\rangle=\frac{1}{t_{\text {tot }}^{A M D}} \sum_{\substack{j=1 \\ \epsilon_{i} \in[q, q+q / 20)}}^{N \text { steps }^{A M D}} \Delta t
$$


where $t_{t o t}^{A M D}$ is the total time spent running AMD (which is accelerated due to the bond-boost potential), and $N$ steps $^{A M D}$ is the number of steps taken after the bond-boost potential is turned on. The time step of $1 \mathrm{fs}$ is used in the sum because at $\epsilon_{i} \geq q$ the bond-boost potential goes to zero and the time is not accelerated.

When the bond-boost potential is activated, the value of $S_{i}$ is initially set to $0.3 \mathrm{eV}$. Once the value of $\left\langle\rho\left(\epsilon_{i}=q\right)\right\rangle$ becomes non-zero the value of $S_{i}$ given by

$$
S_{i}=\max \left(-k_{B} T \ln \left[\frac{\left\langle\rho_{i}\left(\epsilon_{i}=q\right)\right\rangle}{\left\langle\rho_{i}\left(\epsilon_{i}=0\right)\right\rangle}\right]+k_{B} T, \quad 0.3 \mathrm{eV}\right) .
$$

An extra $k_{B} T$ is added to the estimate of the PMF of bond $i$ to increase the acceleration, and a minimum value for $S_{i}$ is set to the initial value of $0.3 \mathrm{eV}$. As stated in Perez et al. 2009, adding extra energy to the PMF estimate will create an energy maximum that may trap the system into exploring a limited region of phase space, lowering the accuracy of the hyperdynamics method. Since the temperature used in this work is quite high $(600 \mathrm{~K}$ is $2 / 3$ of the melting temperature of the material), adding an additional $k_{B} T$ provides a significant increase of $S_{i}$ while creating a maximum in potential energy that is easily overcome by thermal fluctuations. The lower bound of $S_{i}=0.3 \mathrm{eV}$ is chosen because the smallest potential energy barriers calculated previously for this system are around $0.25 \mathrm{eV}$ (so that $0.3 \mathrm{eV}=0.25 \mathrm{eV}+k_{B} T$ ).

\section{Appendix B: Evolving Zone Descriptions}

Since an energy minimization is performed after each point defect is absorbed or emitted from the distributed defect, the defect structure and atomic sites in each zone may change. Since the site concentrations in Zone 4 are not known, it is required that the list of atoms in Zone 4 can grow, but never shrink. Once an atom is included in Zone 4, its site concentration becomes undefined. Likewise, if Zone 4 includes more atoms after the defect evolves, Zone 3 will change to include all atomic sites surrounding the Zone 4 atoms. Since all of these new Zone 3 sites had a defined site concentration at $t^{\prime}$ (they belonged to Zones 1-3), the site concentrations will stay the same, but those sites will be a part of a different zone. Similarly, this process of reassigning the zone number applies to Zones 1-2 as well, while Zone 0 will not change as long as it is far away from the defect.

If this reassignment is necessary due to the defect changing size or shape, the interpolation of Zone 3 site concentrations from the AMD simulations must correspond to the same list of atomic sites in each zone. The Zone 3 site concentrations cannot be updated from AMD values calculated for a different set of atomic sites. Therefore, the original zone configuration at $n$ defects must be remapped to the zone configuration with $n+1$ defects. If the initial zone definitions are created with one or more extra shells of atoms around the distributed defect structure, as done in this work, it is possible that the zone sizes will not change unless the distributed defect grows or moves beyond these extra shells of atoms.

[1] Radu A. Miron and Kristen A. Fichthorn. Accelerated molecular dynamics with the bond-boost method. Journal of Chemical Physics, 119(12):6210-6216, 2003.

[2] Danny Perez, Blas P. Uberuaga, Yunsic Shim, Jacques G. Amar, and Arthur F. Voter. Accelerated molecular dynamics methods: Introduction and recent developments. In Annual Reports in Computational Chemistry, volume 5, chapter 4, pages 79-98. Elsevier, 2009. 
[3] Mads R. Sørensen and Arthur F. Voter. Temperature-accelerated dynamics for simulation of infrequent events. Journal of Chemical Physics, 112:9599-9606, 2000.

[4] B.P. Uberuaga, R.G. Hoagland, A.F. Voter, and S.M. Valone. Direct transformation of vacancy voids to stacking fault tetrahedra. Physical Review Letters, 99:135501, 2007.

[5] Arthur F. Voter. Hyperdynamics: Accelerated molecular dynamics of infrequent events. Physical Review Letters, 78(20):3908-3911, 1997.

[6] Arthur F. Voter. Parallel replica method for dynamics of infrequent events. Physical Review $B$, 57(22):R13985-R13988, 1998.

[7] H. Yoshinaga and S. Morozumi. The solute atmosphere round a moving dislocation and its dragging stress. Philosophical Magazine, 23:1367-1385, 1971.

[8] F. Zhang and W. A. Curtin. Atomistically informed solute drag in al-mg. Modelling Simul. Mater. Sci. Eng., 16:1-18, 2008.

[9] W.A. Curtin and Ronald E. Miller. Atomistic/continuum coupling in computational materials science. Modelling Simul. Mater. Sci. Eng., 11:R33-R68, 2003.

[10] Ronald E. Miller. Direct coupling of atomisticand continuum mechanics in computational materials science. International Journal for Multiscale Computational Engineering, 1:15431649, 2003.

[11] Ronald E. Miller and E.B. Tadmor. A unified framework and performance benchmark of fourteen multiscale atomistic/continuum coupling methods. Modelling Simul. Mater. Sci. Eng., 17:053001, 2009 .

[12] D. L. Olmsted, Rob Phillips, and W. A. Curtin. Modelling diffusion in crystals under high internal stress gradients. Modelling Simul. Mater. Sci. Eng., 12:1-17, 2004.

[13] William H. Press, Saul A. Teukolsky, William T. Vetterling, and Brian P. Flannery. Numerical Recipes: The Art of Scientific Computing (3rd Ed.). Cambridge University Press, 2007.

[14] Arthur F. Voter. A method for accelerating the molecular dynamics simulation of infrequent events. Journal of Chemical Physics, 106(11):4665-4677, 1997.

[15] David A Levin, Yuval Peres, and Elizabeth L Wilmer. Markov Chains and Mixing Times. American Mathematical Society, 2009.

[16] John Price Hirth and Jens Lothe. Theory of Dislocations. John Wiley \& Sons, Inc., 2nd edition, 1982.

[17] Jens Lothe. Theory of dislocation climb in metals. Journal of Applied Physics, 31:1077-1087, 1960.

[18] R.W. Balluffi and R.M. Thomson. Kinetic theory of dislocation climb. ii. steady state edge dislocation climb. Journal of Applied Physics, 33:817-826, 1962.

[19] R.W. Balluffi. Mechanisms of dislocation climb. Physica Status Solidi (b), 31:443-463, 1969. 
[20] R.M. Thomson and R.W. Balluffi. Kinetic theory of dislocation climb. i. general models for edge and screw dislocations. Journal of Applied Physics, 33:803-816, 1962.

[21] Joel Berry, Nikolas Provatas, Jörg Rottler, and Chad Sinclair. Phase field crystal modeling as a unified atomistic approach to defect dynamics. Physical Review B, 89:214117, 2014.

[22] Mukul Kabir, Timothy T. Lau, David Rodney, Sidney Yip, and Krystyn J. Van Vliet. Predicting dislocation climb and creep from explicit atomistic details. Physical Review Letters, 105:095501, 2010.

[23] Timothy T. Lau, Xi Lin, Sidney Yip, and Krystyn J. Van Vliet. Atomistic examination of the unit processes and vacancy-dislocation interaction in dislocation climb. Scripta Materialia, 60:399-402, 2009.

[24] Sanket Sarkar, Ju Li, William T. Cox, Erik Bitzek, Thomas J. Lenosky, and Yunzhi Wang. Finding activation pathway of coupled displacive-diffusional defect processes in atomistics: Dislocation climb in fcc copper. Physical Review B, 86:014115, 2012.

[25] S. J. Plimpton. Fast parallel algorithms for short-ranged molecular dynamics. Journal of Computational Physics, 117:1-19, 1995.

[26] F. Ercolessi and J. B. Adams. Interatomic potentials from 1st-principles calculations - the force-matching method. Europhysics Letters, 26(8):583-588, 1994.

[27] P.B. Hirsch. Extended jogs in dislocations in face-centered cubic materials. Philosophical Magazine, 7:67-93, 1962.

[28] Alan J. Ardell, Howard Reiss, and William D. Nix. Statistics of jogs on dislocations in equilibrium. Journal of Applied Physics, 36:1727-1732, 1965.

[29] K.R. Elder, Mark Katakowski, Mikko Haataja, and Martin Grant. Modeling elasticity in crystal growth. Physical Review Letters, 88:245701, 2002.

[30] Ju Li, Sanket Sarkar, William T. Cox, Thomas J. Lenosky, Erik Bitzek, and Yunzhi Wang. Diffusive molecular dynamics and its application to nanoindentation and sintering. Physical Review B, 84:054103, 2011.

[31] J. Berry, M. Grant, and K.R. Elder. Diffusive atomistic dynamics of edge dislocations in two dimensions. Physical Review E, 73:031609, 2006.

[32] K.R. Elder, Nikolas Provatas, Joel Berry, Peter Stefanovic, and Martin Grant. Phase-field crystal modeling and classical density functional theory of freezing. Physical Review B, 75:064107, 2007.

[33] K.L. Baker and W.A. Curtin. Assessment of phase-field-crystal concepts using long-time molecular dynamics. Physical Review B, 91:014103, 2015.

[34] J. Li. Atomeye: an efficient atomistic configuration viewer. Model. and Simul. in Mater. Sci. and Eng., 11:173-177, 2003. 